\title{
Reversible Cardiotoxicity in a Young Patient Treated with Trastuzumab for Breast Cancer: A Case Report
}

\author{
Ould Amar $N^{1}$, Ben Bachir $H^{1}$, Senhadji $I^{1}$, Bessaih $\mathrm{N}^{2}$ and Toumi $\mathrm{H}^{1^{*}}$ \\ ${ }^{1}$ Service de pharmacovigilance, EHU, Oran, Algeria \\ ${ }^{2}$ Service d'oncologie médicale, EHU, Oran, Algeria
}

\begin{abstract}
We report the case of a young patient with left breast cancer with stage IIB (T2N1M0), negative hormone receptors, HER2-positive and no particular medical history. The patient was particularly sensitive to the neurological and allergic-type toxicity of the adjuvant treatment she received and it required the stopping and successive change of the various prescribed protocols. Trastuzumab was continued as monotherapy for one year; asymptomatic cardiotoxicity was reported following the fall in LVEF (a 17.9\% drop from baseline with a value below 50\%). Active notification was made jointly with the pharmacovigilance team to establish a causal link between the use of trastuzumab and the development of cardiotoxicity. An imputability score calculation was done independently for each product administered subsequently or in combination with trastuzumab and it was concluded that trastuzumab was the most incriminated with a very similar intrinsic score (14). Multidisciplinary care has been taken. Pharmacological treatment and temporary discontinuation of trastuzumab were initiated and trastuzumab was safely restarted after improvement of echocardiographic parameters. A risk management plan and a surveillance strategy for securing trastuzumab in oncology have been proposed.
\end{abstract}

Keywords

Breast cancer, Trastuzumab, Cardiotoxicity, Accountability

\section{Introduction}

Trastuzumab is a humanized monoclonal antibody that selectively binds to the extracellular domain IV on the membrane juxta region of the human epidermal growth factor receptor 2 (HER2) and inhibits the growth of tumor cells in vitro and in vivo via several mechanisms [1]. HER2 is overexpressed in about $15-25 \%$ of all breast cancers [2] and it has been well known that HER2 overexpression is associated with a more aggressive phenotype and a poor prognosis with resistance to certain chemotherapeutic agents [3].

The addition of trastuzumab to adjuvant HER2-positive breast cancer has reduced the risk of relapse and death by approximately $50 \%$ and $33 \%$ [4,5], respectively. As well, the addition of trastuzumab to chemotherapy significantly improved the response rate, the time to progression of the disease, and the reduction in mortality compared to chemotherapy alone in HER2-positive metastatic breast cancer [4].

Generally, trastuzumab is well tolerated, however, cardiotoxicity remains the most debilitating complication that can manifest itself clinically by cardiac insufficiency sometimes requiring discontinuation of treatment and may compromise the vital prognosis of patients. However, unlike anthracycline-induced cardiac toxicity, the toxicity associated with trastuzumab usually responds to standard therapy for heart failure or trastuzumab discontinuation, and there is no evidence that toxicity is related to dose [6].

We present here a case of reversible cardiotoxicity associated with trastuzumab in a woman with breast cancer, an imputability score was established to confirm the causal link.

\section{Case Report}

An active notification was made at the medical oncology department jointly with the pharmacovigilance team, where clinical and therapeutic problems were identified in a 32-year-old woman diagnosed in January 2017 in the service of medical oncology for left breast cancer.

Tissue samples taken from the breast nodule on the left breast revealed histological features of invasive ductal carcinoma. The histochemical immune study showed that the tu-

*Corresponding author: Toumi $\mathrm{H}$, Service de pharmacovigilance, EHU, Oran, Algeria

Accepted: December 02, 2019

Published online: December 04, 2019

Citation: Ould AN, Ben BH, Senhadji I, et al. (2019) Reversible Cardiotoxicity in a Young Patient Treated with Trastuzumab for Breast Cancer: A Case Report. Insights Biomed Res 3(1):86-89 


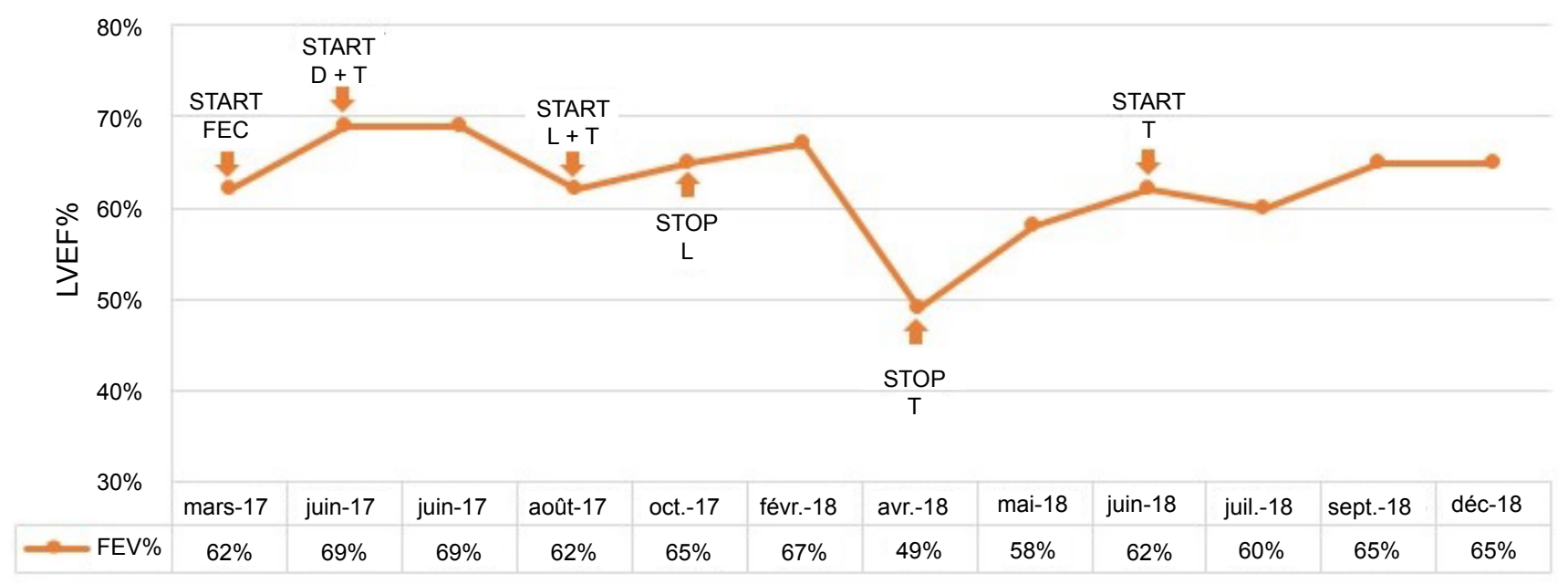

Figure 1: Values of left ventricular ejection fraction during the treatment period.

(FEC: Fluorouracil Epirubicin Cyclophosphamide; D: Docetaxel; T: Trastuzumab; L: Lapatinib).

mor cells were negative for the estrogen and progesterone receptors and positive for the HER2 receptor. The extension assessment is without particularity. It should be noted that the patient had a body mass index of $35.2 \mathrm{Kg} / \mathrm{m}^{2}$ (severe obesity), and a family history of high blood pressure. She was otherwise in a good health with a WHO performance index of 0 and no medical history of heart disease or concomitant medical conditions.

The patient had a total mastectomy with lymph node dissection. The pathological stage of her tumor was IIB (T2N1M0). In March 2017, an adjuvant chemotherapeutic regimen was initiated, based on epirubicin $\left(100 \mathrm{mg} / \mathrm{m}^{2}\right)$, 5-Fluorouracil $\left(500 \mathrm{mg} / \mathrm{m}^{2}\right.$ ) and cyclophosphamide (500 $\mathrm{mg} / \mathrm{m}^{2}$ ) for three cycles repeated every twenty-one day. Cerebellar ataxia and difficulty walking occurred after the second course, the protocol was stopped and replaced by the taxotere $\left(100 \mathrm{mg} / \mathrm{m}^{2}\right.$ every three weeks) associated with trastuzumab $(8 \mathrm{mg} / \mathrm{kg}$ and then $6 \mathrm{mg} / \mathrm{kg}$ every three weeks) in May 2017. However, the patient's condition worsened despite the change in therapy, and a treatment to reduce neuropathic pain was introduced.

In August 2017, after a multidisciplinary consultation meeting, taxotere was stopped and replaced by lapatinib (1000 mg once daily) combined with trastuzumab $(4 \mathrm{mg} /$ $\mathrm{kg}$ followed by $2 \mathrm{mg} / \mathrm{kg}$ every three weeks), a gradual improvement in the patient's condition was noted. Despite the regression of neuropathic disorders, skin rash and acne-like lesions appeared in the patient's face after the third course of lapatinib. In October 2017, it was decided to discontinue lapatinib and to continue trastuzumab $(6 \mathrm{mg} / \mathrm{kg}$ every three weeks) as monotherapy.

It should be noted that serial echocardiography before and during chemotherapy all demonstrated normal left ventricular systolic function (Figure 1). In the period from May to June, abdominal magnetic resonance imaging (MRI) and bone scintigraphy were done revealing hepatic and bone secondary localization respectively.
The patient continues to receive trastuzumab monotherapy for one year, and a regular assessment of cardiac function is made every twelve weeks by Doppler echocardiography. In April 2018 the patient presented cardiotoxicity following an asymptomatic decrease in left ventricular ejection fraction (LVEF) (a fall of $17.9 \%$ compared to the initial value with a value below $50 \%$ ). Trastuzumab was discontinued temporarily and a treatment regimen against heart failure was prescribed, after a month and a half, cardiac function improved (LVEF $=62 \%$ ) and trastuzumab treatment was initiated in June 2018. The patient was receiving trastuzumab monotherapy until January 2019 and no cardiac event was detected.

\section{Discussion}

We report the case of a young patient with breast cancer, with no specific medical history. The patient was particularly sensitive to the neurological and allergic- type toxicity of the adjuvant treatment she received and it required the stopping and successive change of the various protocols prescribed. Trastuzumab was maintained in monotherapy for one year, asymptomatic cardiotoxicity was reported following the fall of the LVEF, and this was reported at the pharmacovigilance service, to prove the causal link between the use of trastuzumab and the onset of cardiotoxicity taking into account the treatment previously given and in combination with trastuzumab.

The calculation of the imputability score was independently established for each drug administered in this patient, based on chronological; semiological and bibliographic criteria (Table 1).

During the imputability study, it was found that all the protocols administered to the patient are potentially cardiotoxic, with a plausible (S2) to likely (S3) semiological score, nevertheless the chronology of the appearance of cardiotoxicity is incompatible with their administration, with the exception of epirubicin (which is an anthracycline) and trastuzumab which are imputed. Trastuzumab has the 
Citation: Ould AN, Ben BH, Senhadji I, et al. (2019) Reversible Cardiotoxicity in a Young Patient Treated with Trastuzumab for Breast Cancer: A Case Report. Insights Biomed Res 3(1):86-89

Table 1: The intrinsic and extrinsic imputability of administered drugs.

\begin{tabular}{|l|l|l|l|l|}
\hline & Chronological score & Semiological score & $\begin{array}{l}\text { Imputability score } \\
\text { (intrinsic score) }\end{array}$ & $\begin{array}{l}\text { Bibliography score } \\
\text { (extrinsic score) }\end{array}$ \\
\hline Fluorouracil & C0 & S2 & I0 & B3 \\
\hline Cyclophosphamide & C0 & S2 & 10 & B3 \\
\hline Epirubicin & C2 & S3 & I3 & B3 \\
\hline Docetaxel & C0 & S2 & B & B2 \\
\hline Lapatinib & C0 & S2 & 10 & B3 \\
\hline Trastuzumab & C3 & S3 & I & \\
\hline
\end{tabular}

C3: Probable; C2: Plausible; C0: Incompatible; S3: Likely; S2: Plausible; 14: Very likely; I3: Likely, I0: Incompatible, B3: Expected effect, B2: Widely published in scientific journals.

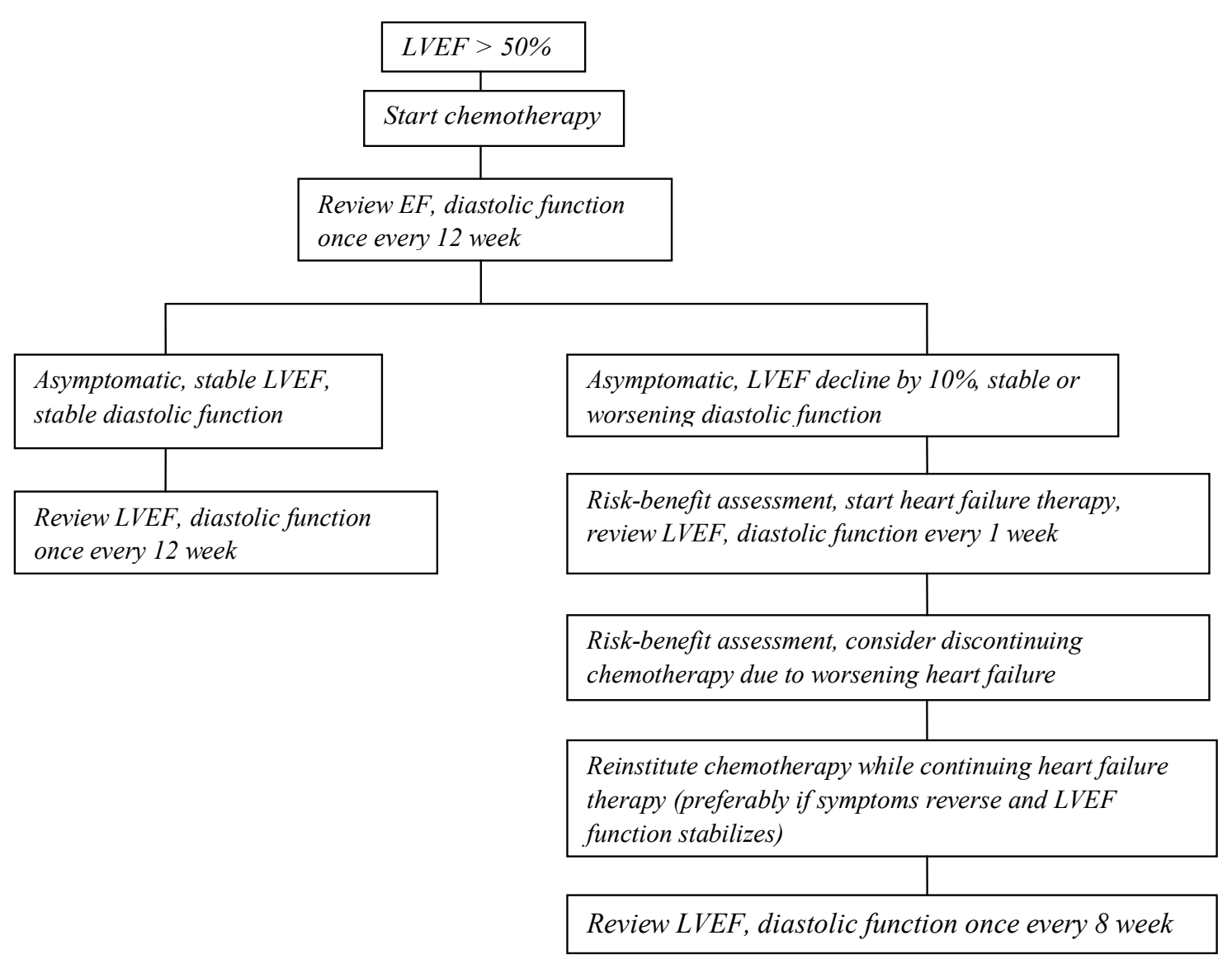

Figure 2: Algorithm for monitoring trastuzumab-induced cardiotoxicity [11].

highest intrinsic score (14) and is implicated in the development of cardiotoxicity in this patient and this is consistent with the literature.

Ewer MS and col report cases of 38 patients with HER2-positive breast cancer who were referred for suspected cardiotoxicity related to trastuzumab. All patients had previously received anthracycline-based chemotherapy. A decrease of more than $10 \%$ in LVEF was observed in all patients after administration of trastuzumab. Temporary discontinuation of trastuzumab was performed and treatment regimen against heart failure was undertaken. An increase in FEV after an average delay of 1.5 months was observed in 37 of the 38 patients. Treatment with tras- tuzumab was resumed in 25 patients [7].

Our case is similar to that of Martin, et al., which report the case of a patient with breast cancer with HER2 positive and negative hormone receptors. The patient received adjuvant chemotherapy with anthracycline followed by trastuzumab, her FEV dropped by $21 \%$. An inhibitor of the conversion enzyme was prescribed and the dose of trastuzumab was reduced. Heart function progressively improved and trastuzumab was resumed without complications [8].

In general, there is a rapid improvement in clinical conditions and ejection fraction after stopping trastuzumab and initiation of a standard regimen that allows trastuzumab to 
be resumed, as was done for our patient.

However, the patient had risk factors that may have a correlative effect on the onset of cardiotoxicity, such as elevated body weight and body mass index, cumulative anthracycline dose, and a family history of cardiovascular disease, a potentially cardiotoxic prior treatment, and the negative status of hormone receptors [9].

Since exposure to trastuzumab and an anthracycline poses the greatest risk of developing cardiotoxicity, the guidelines recommend avoiding simultaneous exposure to both agents. A systematic assessment of cardiac function should precede treatment and careful cardiac monitoring of patients receiving chemotherapy agents should be longstanding. Echocardiogram is the non-invasive choice test for the evaluation of LVEF, contributing to the early detection and rapid management of post-treatment systolic and/or diastolic dysfunction [10].

Recommendations from the literature have been proposed that can be used to monitor cardiac function during trastuzumab therapy (Figure 2).

\section{Conclusion}

Even though, with the limitation of a single case study, current evidence may help to demonstrate the potential reversibility of trastuzumab cardiotoxicity, even after anthracycline use, as well as concomitant administration of docetaxel and lapatinib.

In this case, we deduce an interventional surveillance strategy for securing trastuzumab in oncology through the cardiotoxicity evaluation chart, a risk management plan and pharmacovigilance with an active notification system.

\section{References}

1. Sendur MA, Aksoy S, Altundag K (2013) Cardiotoxicity of novel HER2-targeted therapies. Curr Med Res Opin 29: 1015-1024.

2. Floyd JD, Nguyen DT, Lobins RL, et al. (2005) Cardiotoxicity of cancer therapy. J Clin Oncol 23: 7685-7696.

3. Diasa A, Claudinoa W, Sinhab R, et al. (2016) Human epidermal growth factor antagonists and cardiotoxicity-A short review of the problem and preventative measures. Crit Rev Oncol Hematol 104: 42-51.

4. Romond EH, Perez E, Bryant J, et al. (2005) Trastuzumab plus adjuvant chemotherapy for operable HER 2-positive breast cancer. N Eng J Med 353: 1673-1684.

5. Piccart-Gebhart MJ, Procter M, Leyland-Jones B, et al. (2005) Trastuzumab after adjuvant chemotherapy in HER-2 positive breast cancer. N Eng J Med 353: 1659-1672.

6. D Cardinale, A Colombo, R Torrisi, et al. (2005) Trastuzumab-induced cardiotoxicity: Clinical and prognostic implications of troponin I evaluation. J Clin Oncol 28: 3910-3916.

7. Ewer MS, Vooletich MT, Durand JB, et al. (2005) Reversibility of trastuzumab-related cardiotoxicity: New insights based on clinical course and response to medical treatment. J Clin Oncol 23: 7820-7826.

8. Martins JS, Dos Santos VM, Thommen Teles L, et al. (2012) Reversible cardiotoxicity in a 54-year-old woman treated with trastuzumab. Rev Med Chil 140: 763-766.

9. Huzno J, Les D, Sarzyczny-Slota D, et al. (2013) Cardiac side effects of trastuzumab in breast cancer patients - single centers experiences. Contemp Oncol (pozn) 17: 190-195.

10. Galderisi M, Marra F, Esposito R, et al. (2007) Cancer therapy and cardiotoxicity: The need of serial doppler echocardiography. Cardiovasc Ultrasound 5: 4.

11. Partho P Sengupta, Donald W Northfelt, Federico Gentile, et al. (2008) Trastuzumab-Induced Cardiotoxicity: Heart failure at the crossroads. Mayo Clin Proc 83: 197-203.

DOI: $10.36959 / 584 / 453$

Copyright: (c) 2019 Ould AN, et al. This is an open-access article distributed under the terms of the Creative Commons Attribution License, which permits unrestricted use, distribution, and reproduction in any medium, provided the original author and source are credited. 